\title{
Research on Enterprise Innovation Persistence Patterns Recognition and Selection Based on BP Neural Network
}

\author{
Yutong Ma, Yangyi Meng \\ School of Business Administration, South China University of Technology, Guangzhou, China \\ Email: yutongma0404@163.com
}

How to cite this paper: Ma, Y.T. and Meng, Y.Y. (2019) Research on Enterprise Innovation Persistence Patterns Recognition and Selection Based on BP Neural Network. American Journal of Industrial and Business Management, 9, 658-679. https://doi.org/10.4236/ajibm.2019.93045

Received: March 4, 2019

Accepted: March 19, 2019

Published: March 22, 2019

Copyright $\odot 2019$ by author(s) and Scientific Research Publishing Inc. This work is licensed under the Creative Commons Attribution International License (CC BY 4.0).

http://creativecommons.org/licenses/by/4.0/

(c) (i) Open Access

\begin{abstract}
This paper divided Chinese manufacturing listed enterprises into six innovation persistence patterns according to the innovation persistence and ownership structure, and identified these six patterns by constructing BP neural network model. Under the condition of considering the scale of the enterprise, we made a further analysis on which innovation persistence pattern should be adopted in different growth stages. The results show that small-scale enterprises mainly adopt the centralized continuous innovation pattern and the centralized interval innovation pattern. Medium-scale enterprises mainly adopt the moderate interval innovation pattern. Large-scale enterprises mainly adopt the loose interval innovation pattern. Through the scale classification, we analyzed the business performance of listed enterprises, and we found that the centralized continuous innovation pattern, the centralized interval innovation pattern and the loose continuous innovation pattern are the most effective patterns respectively for small-scale, medium-scale and large-scale enterprises in manufacturing industry. These show that with the expansion of the scale, the ownership structure will be more reasonable; enterprises will put more resources and funds into substantial innovation. With the strong strength of enterprises, the research will be more in-depth and innovative.
\end{abstract}

\section{Keywords}

Innovation Persistence, Ownership Structure, Pattern Recognition, BP Neural Network

\section{Introduction}

In the context of "Industrial 4.0" and cloud computing, big data, Internet of 
things, mobile Internet, smart city and other technologies, R\&D investment has been increasing. $R \& D$ activities have become the core competitiveness that determines whether enterprises can develop healthily and stably and surpass their competitors (Anandarajan et al., 2007). Any long-term innovation activity is inseparable from technological innovation [1] [2] [3] and institutional innovation. Technological innovation has become an important means for enterprises to build core competitiveness and long-term dynamic competitive advantage (Ganter \& Hecker, 2013; Kang \& Baek et al., 2017). Enterprise persistent innovation is the process of continuously introducing and implementing new innovation projects and achieving innovative economic benefits for a long period of time. In China, some enterprises have invention patents [4] [5] with high technical content, but most of the patents are mainly new practical type patents, software copyrights, etc. The patent content has nothing to do with production and operation, and will not bring economic benefits in the future. Some enterprises' patent applications are caused by short-term economic interests or policy-driven. In essence, they do not bring any long-term knowledge accumulation and technological progress in R\&D investment. Therefore, the innovation persistence in this paper will be measured by the number of invention patent applications, and does not include the strategic behavior to cater to government policies or to obtain financial subsidies. The innovation persistence of this paper is to use the number of invention patent applications (Hall \& Harhoff, 2012, Tong et al., 2014) to construct a new variable that can represent innovation persistence for a period of time.

In recent years, the executive compensation of listed enterprises has been widely discussed. An important part of executive compensation is equity incentives. Equity incentives are mainly divided into equity incentives and cash incentives. Equity incentives include stock options, restricted stocks, performance stocks, employee stock ownership plans, etc. Stock option incentive plan uses stocks as a means to motivate operators. Under the authorization of the shareholders' meeting, the board of directors of the enterprise signed an agreement on behalf of the shareholders and the incentive objects led by the operators. When the incentive targets complete certain performance targets or because the performance increases and the stock price rises to a certain extent, the enterprise awards incentives at a certain preferential price. The object stock or a certain price is granted to purchase the stock during the validity period. A restricted stock incentive plan is to reward restricted stock to managers or employees. A listed enterprise grants a certain amount of shares of the enterprise to the incentive target according to predetermined conditions. The incentive object can only sell and benefit from the restricted stock if the working years or performance targets meet the conditions stipulated in the equity incentive plan. Performance stocks refer to a more reasonable performance target at the beginning of the year. If the incentive target reaches the predetermined target by the end of the year, the enterprise grants a certain amount of stock or withdraws a certain incentive fund to purchase the stock. If the performance appraisal fails or the be- 
havior of the enterprise is impaired, abnormal departure, etc., the outstanding stocks of the outstanding shares will be cancelled. Employees own part of the equity by purchasing part of the stock (or equity) and obtain corresponding management rights. The purpose of implementing the employee stock ownership plan is to make the employee become the shareholder. There are two main types. One is that the employees of the enterprise own part of the property rights of the enterprise by purchasing part of the shares of the enterprise and obtain the corresponding management rights. The other is that the employee purchases the entire equity of the enterprise and owns all the property rights of the enterprise, so that the employees have full management and voting rights to the enterprise. Management can obtain a portion of the corresponding real equity from the enterprise in accordance with the incentive policy. Cash incentives include stock appreciation rights, virtual stock rights, virtual stock plans, and profit dividends. But in fact, as shown in Figure 1 (data source is CSMAR database). Restricted stocks, stock options, and stock appreciation rights all belong to equity incentives. As can be seen from the figure, the restricted stock value of manufacturing is about four times that of the second. Stock options are about five times higher than the second place, and stock appreciation rights are higher than all other industries. Therefore, it can be found that more enterprises in the manufacturing industry are willing to choose equity incentives. The reason is that according to the agency theory, when the agent's behavior cannot be fully monitored, if the agent is encouraged by long-term equity, the benefits can be bound together with the interests of shareholders, enabling agents to make active investments in technology innovation based on long-term interests of the enterprise (Jensen \& Meckling, 1976). Especially for business activities with high uncertainty such as $\mathrm{R} \& \mathrm{D}$ investment, the output and potential benefits often have a lag period of several years. Successful innovation behavior is not based on quick success but based on long-term efforts. Therefore, giving the necessary equity incentives to the strategic decision makers and key personnel in the R\&D innovation activities, tying their personal interests to the interests of the enterprise, can prompt them to select the optimal decision based on the long-term perspective and make the necessary commitment and action (Fu, 2012). More importantly, the incentive effect of long-term equity is not only reflected in the convergence of the objective function of executives and shareholders, but also helps the internal management members to maintain unity and cooperation in the R\&D innovation process.

Therefore, in theory, the management's long-term equity incentives have convergence of interest effects in the R\&D innovation practice of the enterprise, which helps the executives to formulate $R \& D$ innovation strategies and strengthen their efforts and teamwork throughout the R\&D innovation process, and reduce laziness and free-riding behavior. In addition, the management's equity incentives also affect the overall structure of the enterprise. The unilateral governance theory based on the "shareholders' interests first" believes that the enterprise is the shareholder's enterprise, the shareholder owns the full ownership 


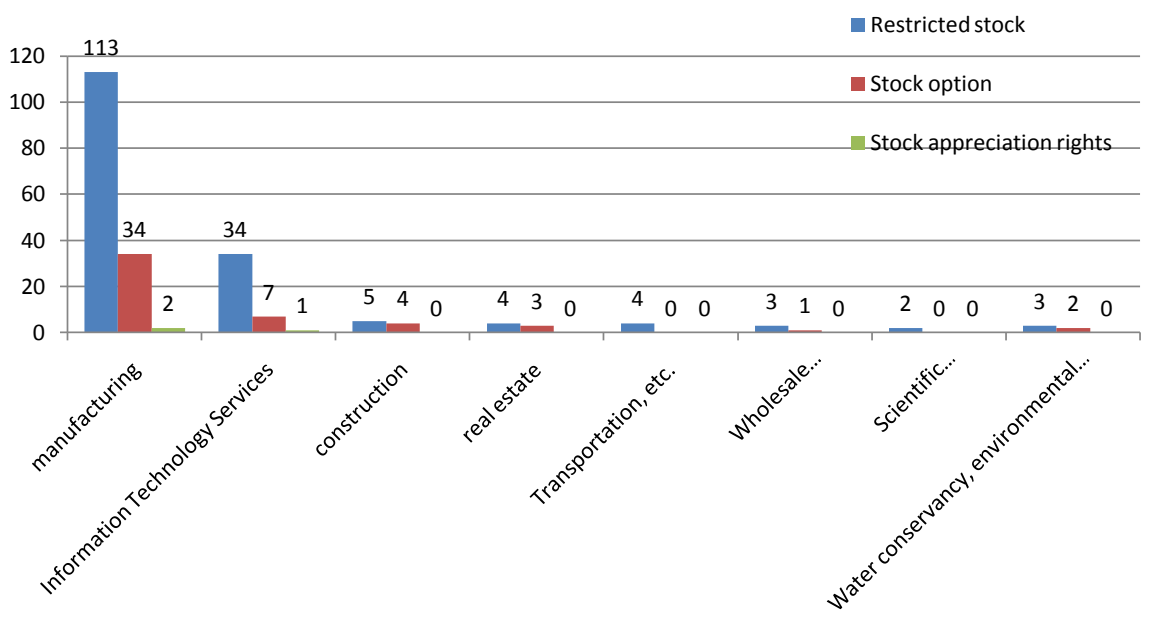

Figure 1. The main selection of incentive tools for various industries. Data Sources: CCER database and CSMAR database.

of the enterprise, and the enterprise's purpose is to maximize the shareholders' interests. Only substantial innovation will bring shareholders a steady stream of benefits, so shareholders pursue corporate innovation and influence innovation behavior.

Jensen and Mecklingz [6] found that with the separation of corporate ownership and management, agency problems began to emerge. The management shareholding ratio is an indicator to measure the degree of separation between ownership and management. Demsetz, Jensen and Meckling [7] claimed the lower the shareholding ratio of management, the higher the separation of ownership and management rights. Shareholders who have separated ownership and management rights cannot directly participate in the enterprise's business activities, but indirectly affect the enterprise's technological innovation through communication and incentives to managers. Francis and Smith [8] found that enterprises with higher shareholdings ( $>30 \%)$ had significantly higher R\&D activities than enterprises with lower management shareholdings $(<15 \%)$, who believed that high equity concentration and shareholder regulation were effective It reduces the agency costs brought to enterprises by $R \& D$ activities. Lerner and Wulf [9] used US enterprises as a sample to find more long-term incentives (such as stock options or restricted stocks) that lead to more R\&D output (such as higher patent citation rates), but short-term Incentives had nothing to do with the enterprise's R\&D output. However, the study sample is small and contains only 141 enterprises.

In summary, the current research on Innovation persistence has been extensive. However, the behavioral characteristics of an enterprise are often the result of a combination of factors. From the above, it can be seen that the degree of technological innovation of enterprises with different ownership structures [10] [11] [12] [13] is also different. There is currently only a small amount of literature discussing the impact of ownership structure on R\&D intensity and R\&D output. Therefore, this paper proposes a new method for dividing the innovation 
persistence pattern of manufacturing enterprises based on the ownership structure of listed enterprises in China's manufacturing industry. This paper uses BP neural network model, and discusses the recognition effect of BP neural network on these patterns and explains the significance of this pattern division.

Therefore, the paper is divided into four parts. The first part introduces the theoretical basis of this paper. The second part mainly introduces the definition of pattern recognition, the method of BP neural network. The third part mainly uses BP neural network to construct a recognition model for six innovation persistence patterns of China's manufacturing enterprises, and verify the recognition effect. The fourth part mainly studies the evolution of the enterprise pattern with the development of the enterprise scale through descriptive statistics, and further explores the difference in business performance of different innovation patterns under different enterprise scales. It can provide guidance for the innovation path of China's manufacturing industry enterprises.

\section{Research Methods and Model Design}

\subsection{Patterns Recognition Methods}

Pattern recognition [14] method is a mathematical statistical method for processing information and classifying information by means of computer. The first step in applying the pattern recognition method is to create a pattern space. The pattern space refers to the multi-dimensional space formed by many indicators that affect the target when examining an objective phenomenon. Each indicator represents a pattern parameter. Suppose a phenomenon consists of several events (samples), each event has $\mathrm{P}$ characteristic parameters $\left(x_{1}, x_{2}, \cdots, x_{p}\right)$, which form a P-dimensional pattern space. The characteristic parameters of each event represent a pattern. Pattern recognition is to analyze the distribution characteristics of various patterns in multidimensional space, divide the pattern space, and identify the clustering of various patterns to make judgments or decisions. The analysis method utilizes the "mapping" and "inverse mapping" techniques. Mapping refers to transforming a multidimensional mode space into a two-dimensional plane in which all patterns (sample points) of the multidimensional space are projected. In a two-dimensional plane, different categories of patterns are distributed between different regions with distinct boundaries. This determines the direction of optimization back to the multidimensional space (primitive space), to obtain real information, to help people find out the rules or make decisions, to guide actual work or experimental research.

The current mainstream technical methods are statistical pattern recognition method [15] [16], structural pattern recognition method [17] [18] [19], fuzzy pattern recognition method [20] [21] and neural network pattern recognition method [22]-[27]. Statistical pattern recognition widely samples the identified objects. A statistical distribution model of different kinds of attribute features is established, and the probability that it belongs to different kinds is calculated according to the characteristics of the target at the time of recognition. Then, 
according to the probability, the target is judged as the risk value corresponding to each category, and the risk value is selected as the target category. Structural pattern recognition identifies a description of the structural features of the object to be identified. It treats an identified object as a language structure. Fuzzy pattern recognition is based on the thinking logic of human identification of things, drawing on the recognition characteristics of human brain, turning the binary logic commonly used in computers to continuous logic, and using fuzzy information to classify patterns. Bring a computer or machine with intelligence that is close to humans. Neural network pattern recognition is to correctly map the pattern from the feature space to the class space, or to implement the class division in the feature space. The difficulty of pattern recognition is closely related to the distribution in the pattern and feature space, if any two classes in the feature space can be distinguished by a hyperplane. Then the pattern is linear and separable, and the recognition at this time is easier.

After years of research and development, pattern recognition technology has been widely used in many important fields such as artificial intelligence, computer engineering, machine science, neurobiology, medicine, detective science and high energy physics, archaeology, geological exploration, aerospace science and weapon technology. Such as speech recognition, speech translation, face recognition, fingerprint recognition, recognition of handwritten characters, industrial fault detection, precision guidance, etc. The rapid development and application of pattern recognition technology has greatly promoted the national economic construction and the modernization of national defense technology. Because neural network pattern recognition can deal with some environmental information is very complicated, the background knowledge is not clear, and the inference rules are not clear. Therefore, this paper selects the neural network recognition model with better overall performance as the pattern recognition method.

\subsection{BP Neural Network Model Design}

This paper uses BP neural network model. In the 1940s, McCulloch and Pitts [28] first proposed the neuron MP model, which modeled the model of biological neuron processing, and was the basis for the development of subsequent neural networks. Later, Rosenblatt et al. [29] improved on the basis of the MP model, proposed and enhanced the learning function, enabling the model to perform pattern recognition. Hopfield [30], Rumelhart [31] and other scholars have proposed many improved neural network models. Until the 1980s, Sejnowski [32], Werbos [33], Rumelhart [34] and others proposed new concepts such as "hidden unit", BP algorithm for neural network learning, multi-layer feed forward network, etc., which made the neural network further developed. Up to today, a variety of neural networks, such as convolutional neural networks, BP neural networks, radial-based neural networks, etc., which have their own characteristics and are widely used. 
At present, most of the research on the direction of innovation persistence adopts linear regression or probit nonlinear regression model. Their advantage lies in the obvious revealing of the relationship between independent variables and dependent variables, but the disadvantage is that this model cannot be used to achieve more complex operations, such as pattern recognition, in fact the relationship between independent variables and dependent variables is often a complex nonlinear model which is difficult to visually express. The BP neural network is a black box process and has a non-linear mapping capability without the need to determine the mapping relationship in advance. Internally, it trains data, learns the rules, and finally obtains the predicted output with the smallest mean square error of the actual output value. It has the advantages of relatively short model fitting time, self-learning, self-adaptation, and fault tolerance. After decades of development, BP neural network has become one of the models with wide application and significant classification effect. Therefore, BP neural network is used to identify and analyze patterns in our research.

The Back Propagation (BP) network model is a multi-layer feed forward neural network trained according to the error back propagation algorithm. The topology is mainly divided into input layer, hide layer and output layer as shown in Figure 2. BP neural network is divided into two processes: 1) working signal forward transfer sub-process; 2) error signal reverse transfer sub-process. In a BP neural network, a sample has $\mathrm{n}$ inputs, $\mathrm{m}$ outputs, and there are usually several hidden layers between the input layer and the output layer.

In the BP neural network, the neurons in the upper and lower network layers are fully connected, and there is no connection between the neurons in the same layer. The basic BP algorithm includes two processes, forward propagation of signals and back propagation of errors. In the forward propagation, the input signal acts on the output node through the hidden layer, and generates an output signal through nonlinear transformation. If the model output does not match the expected output, the error propagation process is performed. Error back propagation is to pass the output error back to the input layer through the hidden layer, and distribute the error to all the nodes in each layer. The error signals obtained by each layer are used as the basis to adjust the weight of each node. By adjusting the strength of the connection between the nodes of each layer and the threshold, the error is reduced along the gradient direction. After repeated learning and training, when the network parameters (weights and thresholds) corresponding to the minimum error are found, the training stops. At this time, the trained neural network can process the input information of similar samples and output the information with the least error of the nonlinear transformation.

Neural network pattern recognition model has many advantages: 1) It has self-organization and self-learning ability, can directly input data and learn. Some networks can also adaptively adjust the structure of the network. The neural network classifier also has the functions of pattern transformation and pattern feature extraction. The neural network does not need to make any 


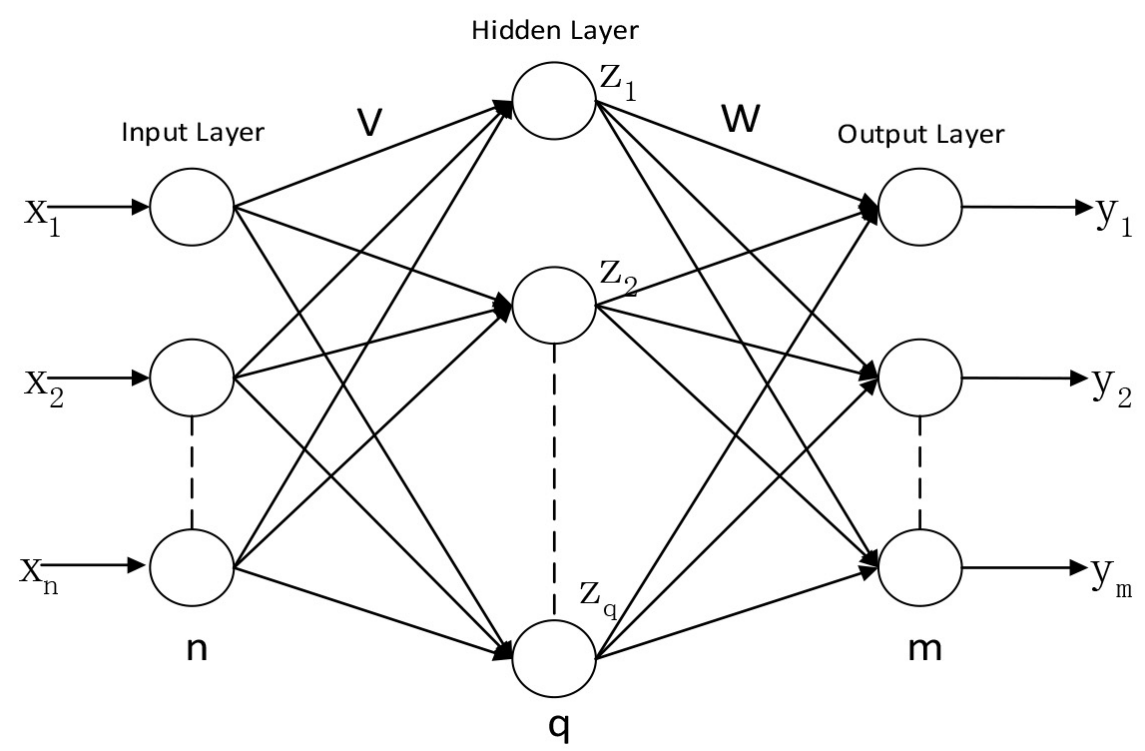

Figure 2. Three-layer neural network topology.

assumptions about the distribution state of the objects to be processed in the sample space, but learns the relationship between the samples directly from the data, so they can also solve the identification problems that cannot be solved because the sample distribution is unknown. 2) The neural network has the ability to promote. It can correctly process data similar to the original training samples based on the similarity between samples. 3) The network is non-linear, it can find complex interactions between system input variables. In a linear system, changing the input tends to produce a proportional output. But in a nonlinear system. This influence relationship is a high-order function. This feature is well suited for real-time systems because real-time systems are often non-linear. Neural networks provide a practical solution to this complex system. 4) Neural networks are highly parallel, that is, a large number of similar or independent operations can be performed simultaneously. This parallelism makes it thousands of times faster than traditional microprocessors and digital signal processors when dealing with problems, which increases the processing speed of the system and provides the necessary conditions for real-time processing.

Based on the above advantages, neural network pattern recognition has developed into an important method in the field of pattern recognition. It plays an irreplaceable role in traditional pattern recognition methods.

In this paper, the BP neural network model is adopted for the specific pattern recognition of the listed enterprises in the manufacturing industry. The model design is shown in Figure 3. Firstly, the original data of listed manufacturing enterprises is conducted to data pre-processing, and construct the input layer and output layer of the BP neural network model for the innovation persistence pattern recognition of manufacturing enterprises. Then put the input data and the expected output data into BP neural network model, set the network topology, random weights, thresholds, and obtain the model that used to recognize 


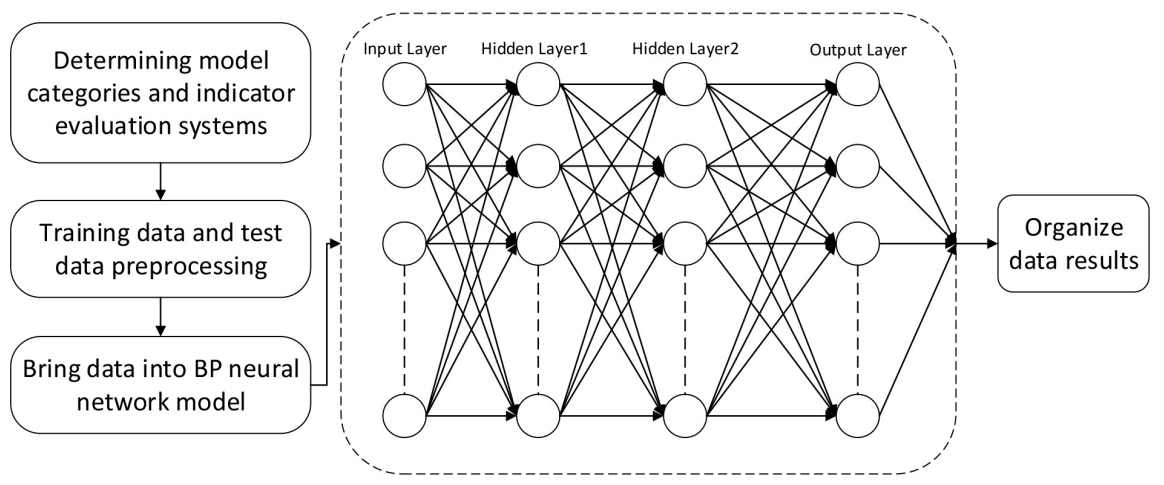

Figure 3. BP neural network model design.

patterns after training. Next, randomly set different network structures and weight threshold repetition training to obtain different models. When the model performed well (the error is less than the given value), the training is stopped. We recorded the weight threshold and topology at this time, and completed the construction of the innovative persistence patterns recognition model.

\subsection{Enterprise Innovation Persistence Pattern}

In order to judge the specific pattern of manufacturing listed enterprises, this paper is based on the ownership structure and Innovation persistence of enterprises. The ownership structure mentioned in this article refers to the proportion of shares of different nature in the total share capital of the joint stock enterprise and their mutual relationship. Equity is a right that can be claimed against an enterprise based on its status (identity).

The ownership structure mentioned in this article refers to a kind of innovation behavior. In the past three years, if there are at least two invention patents per year for two or more years, then we call this situation a continuous innovation. In the past three years, if there are at least two invention patents per year for two or more years, then we call this situation continuous innovation. In the past three years, if there is only one year, the enterprise has patented inventions, or has not produced invention patents for three years, then we call this situation interval innovation. The innovation persistence is taken as the ordinate and the ownership structure is taken as the abscissa. In the light of the relevant literature, the ordinate is divided into two categories, continuous innovation and interval innovation, and the abscissa is divided into three categories, centralized equity, moderate equity and loose equity. Combine these two dimensions, and finally get six different innovative persistence patterns with different distinct characteristics are obtained, as shown in Figure 4. We call these six patterns the centralized continuous innovation pattern, the centralized interval innovation pattern, the moderate continuous innovation pattern, the moderate interval innovation pattern, the loose continuous innovation pattern, the loose interval innovation pattern.

Among the six patterns, the characteristics of the enterprises represented by the loose continuous innovation pattern are that the enterprises are relatively 


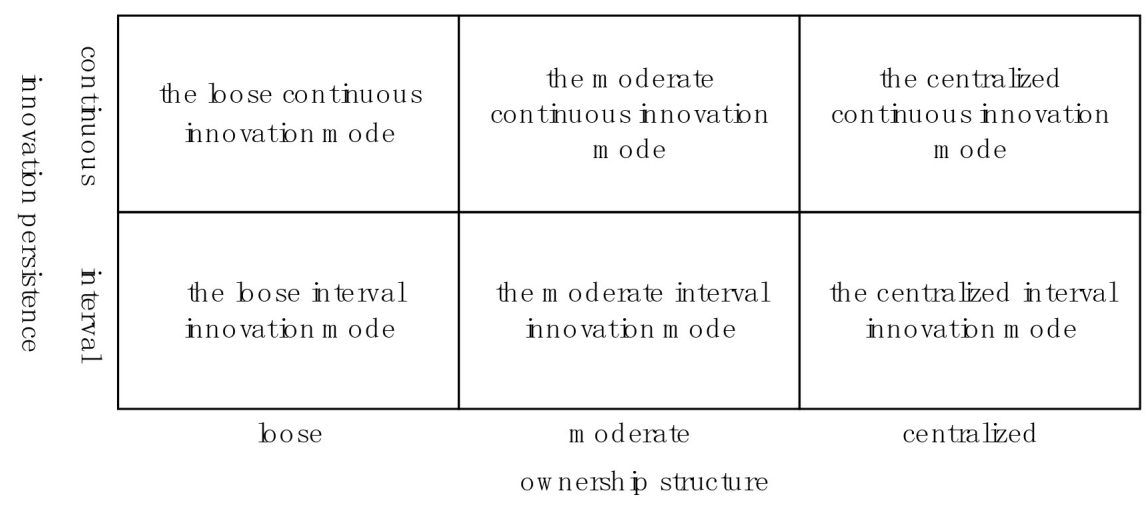

Figure 4. Innovation persistence patterns of China's listed manufacturing enterprises.

mature, the ownership structure is relatively scattered, and there are sustained substantial innovation outputs within three years. Enterprises with such characteristics generally have a large scale. The characteristics of the enterprises represented by the loose interval innovation pattern are that the ownership structure is more dispersed and the substantial innovation is intermittent. The characteristics of the enterprises represented by the moderate continuous innovation pattern are that the ownership structure is relatively dispersed and capable of continuous innovation. The characteristics of the enterprises represented by the moderate interval innovation pattern are that the ownership structure is relatively dispersed, and innovation is intermittent. The characteristics of the enterprises represented by the centralized continuous innovation pattern are that the ownership structure is concentrated, mostly family businesses or equity is concentrated in the hands of management, and has continuous innovation capabilities. The characteristics of the enterprises represented by the centralized interval innovation pattern are that the ownership structure is concentrated, but they cannot have continuous innovation outputs due to limited investment capacity.

The distribution of these six patterns of enterprises in the sample data is not uniform. According to the sample size, the enterprises belong to the moderate continuous innovation pattern and the loose continuous innovation pattern are relatively less.

\section{Innovation Persistence Pattern Recognition}

In this paper, we used BP neural network to identify the six manufacturing enterprise innovation persistence patterns. The neural network model is constructed by designing model feature items, network topology and parameters. Through continuous training and comparison, the optimal BP neural network recognition model was selected. After then, we also tested and verified the effect of the model.

\subsection{Evaluation Index System for Innovative Persistence Patterns}

In the BP neural network model, the input layer data structure plays a crucial 
role in the model results. The stronger the relationship between the data feature of the input layer and the dependent variable, the better the recognition effect of the model. To this end, we designed the following enterprise pattern recognition index system to finish feature selection [35] [36] [37] [38], as shown in Table 1.

First of all, the innovation persistence of manufacturing enterprises is mainly divided into two first-level indicators, ownership structure and innovation persistence. Among them, the ownership structure is mainly divided into five second-level indicators. Circulating stock proportion, the shareholding ratio of institutional investors, remuneration of the management, remuneration of the board of directors, and remuneration of the board of supervisors. Innovation persistence is mainly divided into twelve second-level indicators. Invention patent, invention patent persistence, invention patent proportion, non-invention patent proportion, enterprise size, enterprise age, $R \& D$ investment, $R \& D$ intensity, ROA, asset-liability ratio, quick Ratio, operating cash flow.

This evaluation system comprehensively describes the enterprise's situation from the aspects of the structure, internal and external factors of the enterprise. Thus makes a comprehensive and accurate assessment of the enterprises' innovation persistence.

\subsection{Data Collection and Data Preprocessing}

After comparing the enterprises' innovation data published by different websites, we selected CCER database and CSMAR database with reliable data sources. We manually collected the initial data of the corresponding indicators. The research object of this paper is the enterprises that can collect data among the listed enterprises in the A-share manufacturing industry in Shanghai and Shenzhen in 2016. The sample screening process is as follows: First, delete the listed enterprises of ST and PT and the enterprises that conducted IPOs that year. Second, delete the total assets and the owners whose equity is negative or zero in the remaining enterprises. Third, delete the main research variable data missing or abnormal value samples in the remaining enterprises. We final used 1805 enterprises' data samples from 2007 to 2016 as the original data. In order to ensure the quality of the data, data preprocessing is required before the data is brought into the model. First, we dealt with the vacancy value. There were only a few vacancy records. In order to avoid the impact of the vacancy value, the vacancy value was set to the enterprise average value. Secondly, the original data was converted to data items in the index system according to the calculation formula. The data collected in this paper are quantitative indicators, so the calculation formula can be directly used to generate the required feature items of the model. At the same time, because each feature has different dimensions, log function conversion is performed on some feature items with large dimensions. The min-max standardization process is performed on all feature items. The conversion function is

$$
x_{i j}^{\prime}=\left(x_{i j}-\min _{j} x_{i j}\right) /\left(\max _{j} x_{i j}-\min _{j} x_{i j}\right)
$$


Table 1. Evaluation index system for innovation persistence pattern.

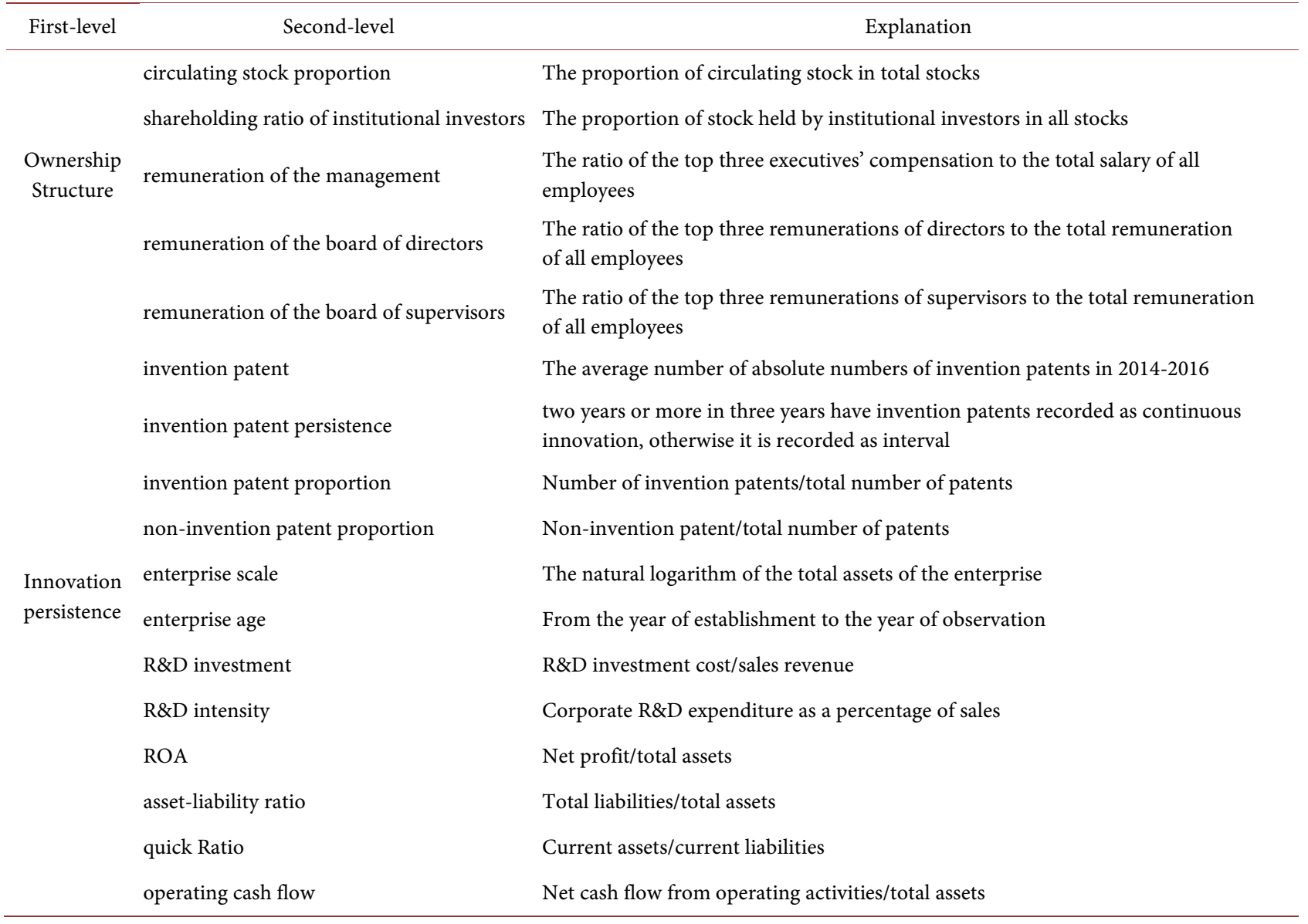

$x_{i j}$ is the feature item $j$ of enterprise $I, x_{i j}^{\prime}$ is the score after $x_{i j}$ is standardized.

\subsection{Topology of BP Neural Network}

We hope to use the BP neural network model in Matlab to study the training data well, and have a good recognition effect on the six Innovation persistence patterns of manufacturing enterprises. Therefore, it is necessary to determine the $\mathrm{BP}$ neural network topology. For the number of hidden layers, according to experience, 1,2 , and 3 are selected respectively, and the results showed that the BP neural network structure with two of hidden layers has better model effect. For the determination of the number of nodes in each layer, first we tried to take points in a larger interval. For example, the number of nodes in each layer tried $1,10,20,30$ respectively. Secondly, we tried different node numbers between the better performing number of nodes in first step (this paper was the interval of 1 10), and used Monte Carlo method to select the average best performing hidden layer structure in average. As shown in Figure 5, a four-layer BP neural network structure is finally formed. The number of nodes in the input layer is 17 , the number of nodes in the first layer of the hidden layer is 4 , and the number of nodes in the second layer of the hidden layer is 10 , and the nodes in the output 
Neural Network

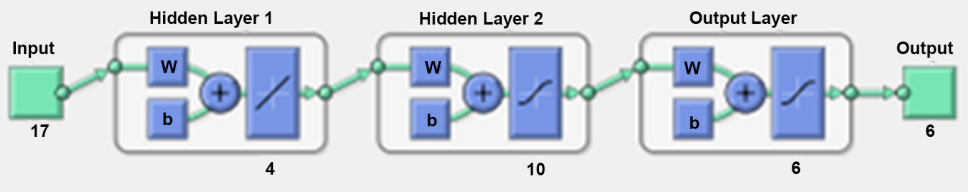

Figure 5. BP neural network topology.

layer is 6. Noted that the prediction results of one enterprise in BP neural network are the possibilities of each pattern, which are six decimal values between 0 - 1 . So we selected the largest of the six decimal values and set it to 1 , and the other values we set them to 0 , which means we chose the most likely pattern as the final forecast pattern for this enterprise.

\subsection{Training and Testing of BP Neural Network}

We collected a total of 1805 enterprises' data. We randomly divided data into training set and test set according to the ratio of 4:1, in the meanwhile ensuring that the training set was the same as the proportion of each pattern in the test set. Therefore, this paper used 1444 data as training data and 361 data as test data. Using the BP neural network structure constructed above, different combinations of network parameters were tried on this basis. After comparing the experimental results, the transfer function of each layer was finally set to purelin, tansig, tansig, purelin, the training function was set to trainlm, the learning function was set to traingda, the learning rate was set to 0.05 . This combination was a relatively small combination of parameters with a root mean square error. Using the above topology and parameters, the training data was brought into the model for learning. It is important to note that the weights and thresholds of the BP neural network in Matlab were randomly initialized, so the results of each training model were different. We used the same network structure to learn multiple times, and selected the best performing BP neural network training model (the model result with the smallest global convergence error), and used this model as the final BP neural network model.

After constructing the model, we performed data preprocessing on the test data, and used the trained BP neural network model to verify the simulation. The real value of each enterprise in the test set presents the real pattern, and the predicted value of each enterprise in the test set presents the predicted pattern obtained by using the trained BP neural network model. As shown in Table 2, the predicted value of each enterprise is represented by vectors. $(100000)^{\mathrm{T}}$ presents the loose continuous innovation pattern, $(010000)^{\mathrm{T}}$ presents the loose interval innovation pattern, $(001000)^{\mathrm{T}}$ presents centralized continuous innovation pattern, $(000100)^{\mathrm{T}}$ presents the moderate interval innovation pattern, $(000010)^{\mathrm{T}}$ presents the moderate continuous innovation pattern, and $(000001)^{\mathrm{T}}$ presents the centralized interval innovation pattern. The overall forecast accuracy rate has reached a high level about $89 \%$. 
Table 2. Example of the expected output and actual output value of the neural network model.

\begin{tabular}{cll}
\hline Enterprise number & Real value & Predicted value \\
\hline Enterprise 05 & $(100000)^{\mathrm{T}}$ & $(100000)^{\mathrm{T}}$ \\
Enterprise 31 & $(010000)^{\mathrm{T}}$ & $(100000)^{\mathrm{T}}$ \\
Enterprise 89 & $(100000)^{\mathrm{T}}$ & $(100000)^{\mathrm{T}}$ \\
Enterprise 104 & $(001000)^{\mathrm{T}}$ & $(001000)^{\mathrm{T}}$ \\
Enterprise 153 & $(000100)^{\mathrm{T}}$ & $(000010)^{\mathrm{T}}$ \\
Enterprise 162 & $(000010)^{\mathrm{T}}$ & $(000010)^{\mathrm{T}}$ \\
Enterprise 236 & $(010000)^{\mathrm{T}}$ & $(010000)^{\mathrm{T}}$ \\
Enterprise 248 & $(000100)^{\mathrm{T}}$ & $(000100)^{\mathrm{T}}$ \\
Enterprise 269 & $(000010)^{\mathrm{T}}$ & $(000010)^{\mathrm{T}}$ \\
Enterprise 287 & $(000010)^{\mathrm{T}}$ & $(000010)^{\mathrm{T}}$ \\
Enterprise 302 & $(000001)^{\mathrm{T}}$ & $(000001)^{\mathrm{T}}$ \\
Enterprise 306 & $(000001)^{\mathrm{T}}$ & $(000001)^{\mathrm{T}}$ \\
Enterprise 459 & $(100000)^{\mathrm{T}}$ & $(100000)^{\mathrm{T}}$ \\
Enterprise 490 & $(001000)^{\mathrm{T}}$ & $(001000)^{\mathrm{T}}$ \\
Enterprise 526 & $(000001)^{\mathrm{T}}$ & $(000001)^{\mathrm{T}}$ \\
Enterprise 531 & $(010000)^{\mathrm{T}}$ & $(010000)^{\mathrm{T}}$ \\
\hline
\end{tabular}

\section{Innovation Persistence Pattern Selection}

After ensuring the BP neural network can effectively identify the patterns, this paper attempted to further analyze the changing trend of the pattern in the process of enterprise growth according to the characteristics of the enterprise pattern in different stages of development. The results can help manufacturing enterprises to better position themselves and provide advice on the optimal allocation of resources.

\subsection{The Evolution of Innovation Persistence Pattern}

The development of manufacturing enterprises has obvious stages. Small-scale enterprises are in the growth stage, medium-scale enterprises are in the development stage, and large-scale enterprises are in the mature stage. This paper first classified the enterprises in the sample according to the scale of the enterprise and divided them into small-scale enterprises, medium-scale enterprises and large-scale enterprises. In order to verify the rationality of the classification, we did descriptive statistical analysis of the data for small-scale, medium-scale and large-scale enterprises which is shown in Figure 6.

It can be clearly seen that small-scale, medium-scale and large-scale enterprises have significant differences in key indicators. We have counted four indicators. They are institutional shareholdings ratio, financing constraints, fixed assets and quick ratios. The average shareholding ratio of small-scale enterprises 


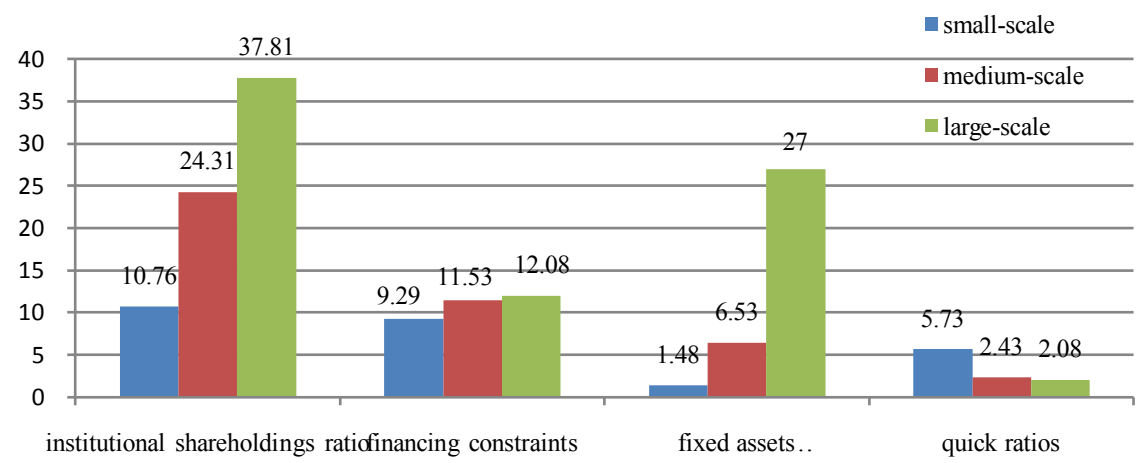

Figure 6. Comparison of characteristics of manufacturing enterprises of different scales. Data Sources: CCER database and CSMAR database.

is $10.76 \%$, that of medium-scale enterprises is $24.31 \%$, and that of large-scale enterprises is $37.81 \%$. As the size of the enterprise increases, the proportion of institutional holdings gradually increases. It can be seen that the three types of enterprise data form a clear gradient, indicating that when the enterprise grows from small-scale to large-scale, there are more institutional investors to invest, and the equity will be further dispersed. The average financing constraint for small-scale enterprises is 9.29 , the average for medium-scale enterprises is 11.53, and the average for large-scale enterprises is 12.08. It can be seen that when the enterprise grows from small-scale to large-scale, the financing constraints also grow from small to large. This is in line with the current reality, because as manufacturing enterprise continues to expand, it will continue to increase funding for research and development and operations. But innovation output is not a one-off event. Therefore, in the process of expanding the scale of the enterprise, on the one hand, the capital market itself has insufficient attention to the manufacturing industry, and on the other hand, the amount of R\&D investment is enormous, which leads to the increasing financing constraints of enterprise. The average fixed assets of small-scale enterprises is 148 million yuan, the average for medium-scale enterprises is 653 million yuan, and the average for large-scale enterprises is 2.7 billion yuan. The characteristics of the industry determine that manufacturing enterprise needs a large amount of fixed assets, so it can be clearly seen that with the expansion of the scale of enterprise, fixed assets show a clear growth trend. The average quick ratio of small-scale enterprises is $5.73 \%$, medium-scale companies average $2.43 \%$, and large-scale enterprises are $2.08 \%$. It can be seen that all types of enterprises have certain solvency, although the short-term solvency of small-scale enterprises will be significantly higher than that of large-scale enterprises, it also shows that the utilization rate of small-scale enterprises is low. Overall, such classification is reasonable.

After the scale division, we explored the trends in the pattern of manufacturing enterprises from small-scale to large-scale. This will help enterprises to achieve better transformation and upgrading. Based on the enterprises' ownership structure and innovation persistence characteristics, the scatter plot is shown in Figure 7. The scatter plots generally show a V-shaped distribution. 


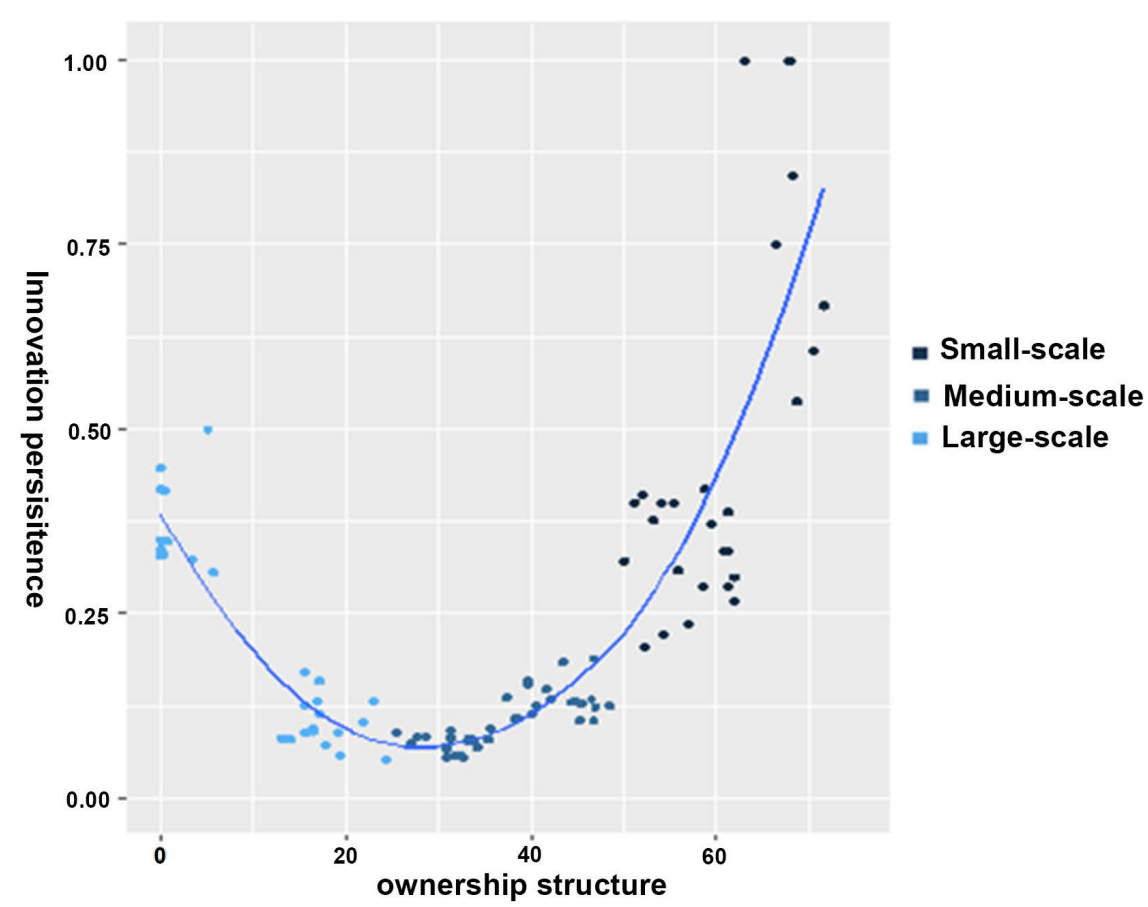

Figure 7. Evolution trend of enterprise pattern. Data Sources: CCER database and CSMAR database.

Small-scale enterprises are mainly the centralized interval innovation pattern and the centralized continuous innovation pattern. Medium-scale enterprises are mainly the moderate interval innovation pattern. Large-scale enterprises are mainly the loose interval innovation pattern, and innovative behavior tries to gradually develop from interval to continuous.

It can be concluded that as the scale of manufacturing enterprises continues to expand, equity becomes more dispersed, ownership and management rights begin to gradually separate. In the small enterprise stage, the innovation persistence is mostly developed from continuous to interval. This is because in the early stage, small enterprises have carried out innovative activities, but in order to maintain the stability of cash flow, they mainly carry out innovations in application or appearance. R\&D investment is relatively small and profits are increased. But in the long run, it is difficult for products to survive in the market without unique technical support. So when the scale of small enterprises increases, enterprises will have a certain amount of funds, they are more willing to spend a lot of time and research investment to make substantial innovations. The innovative output of such research is not inevitable, and may appear together, so the innovation will show the characteristics of the interval in a long time. It can be seen that the continuous trend of innovation for medium-sized enterprises has indeed decreased first and then increased slightly. While becoming large-scale enterprises, with the enhancement of the foundation of innovation research, the innovation capability is continuously improved, then the innovation output interval tends to be narrowed. 


\subsection{Performance of Innovation Persistence Pattern in Different Enterprise Scales}

Although the above analysis illustrates the trend of enterprise pattern selection at different times, these patterns do not represent the most efficient pattern for the corresponding enterprise scale. In order to analyze which pattern has the highest efficiency, this paper used the performance level to analyze. We chose scholarly-accepted EBIT to represent the performance level of the enterprise.

In Figures 8-10, the average business performance of enterprises in each pattern under different enterprise scales is calculated and plotted. We can see the rankings of the business performance of small-scale enterprises from high to low are the centralized continuous innovation pattern, the centralized interval innovation pattern, the loose interval innovation pattern and the moderate interval innovation pattern. The rankings of the business performance of medium-scale enterprises from high to low are the centralized interval innovation pattern, the loose interval innovation pattern, the moderate interval innovation pattern, the moderate continuous innovation mode and the centralized continuous innovation pattern. The rankings of the business performance of large-scale enterprises from high to low are the loose continuous innovation pattern, the moderate continuous innovation pattern, the centralized interval innovation pattern, the centralized continuous innovation pattern, the loose interval innovation pattern and the moderate interval innovation pattern. In summary, the centralized continuous innovation pattern, the centralized interval innovation pattern, the loose continuous innovation pattern are the best business performances respectively for small-scale, medium-scale and large-scale enterprises.

\section{Conclusions}

Using of BP neural network model, we discussed the identification and selection of innovation persistence patterns of listed enterprises in China's manufacturing industry, and obtained the following important conclusions.

Firstly, the evaluation index system is used as the input vector, and the enterprise innovation persistence pattern is used as the output vector. The trained BP neural network model can correctly and reasonably identify the enterprises' innovation persistence patterns.

Secondly, after dividing the scale of the enterprise, we studied the evolution of enterprise innovation persistence pattern, and the overall trend is V-shaped. Small-scale enterprises are mainly the centralized interval innovation pattern and the centralized continuous innovation pattern. Medium-scale enterprises are mainly the moderate interval innovation pattern, while large-scale enterprises are mainly the loose interval innovation pattern. With the continuous scale growth of enterprises, the equity has been concentrated to relatively dispersed, which has increased the ability of enterprises to resist risks. The level of innovation and ability is constantly improving, and enterprise innovation is transforming into basic R\&D activities. In an attempt to develop more technological 


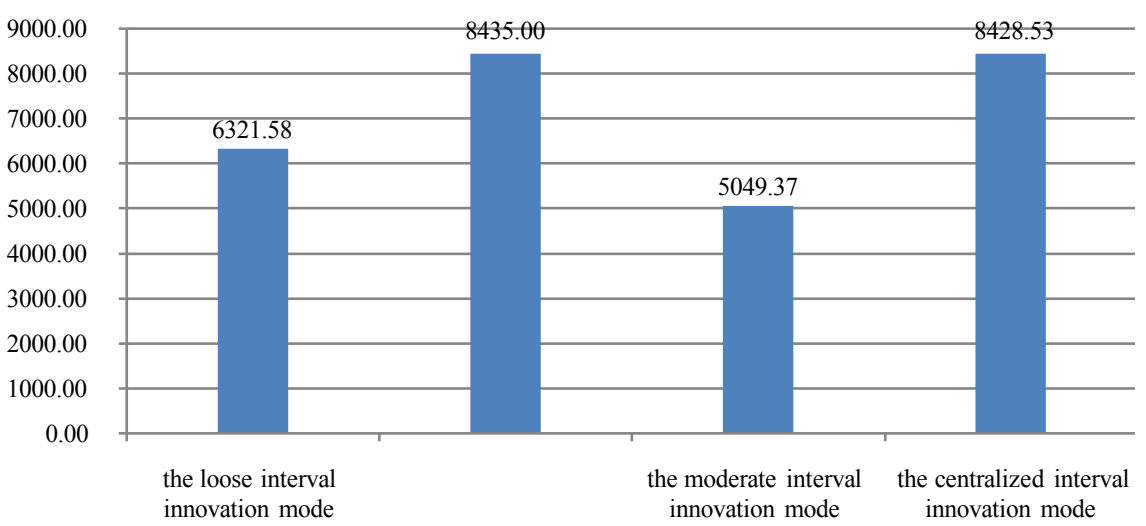

Figure 8. Business performance of different patterns of small-scale enterprises. Data Sources: CCER database and CSMAR database.

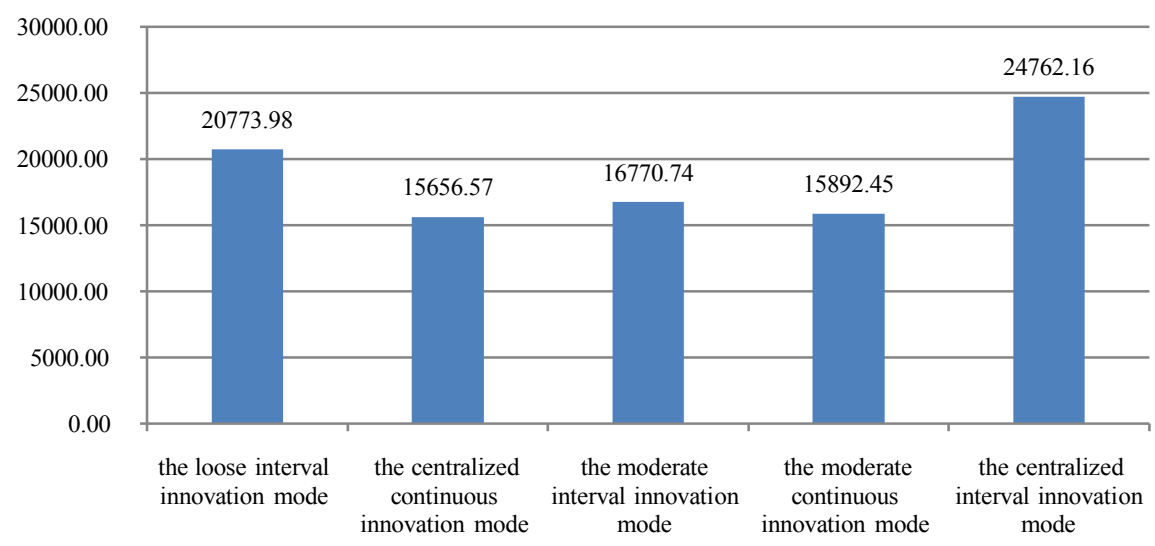

Figure 9. Business performance of different patterns of medium-scale enterprises. Data Sources: CCER database and CSMAR database.

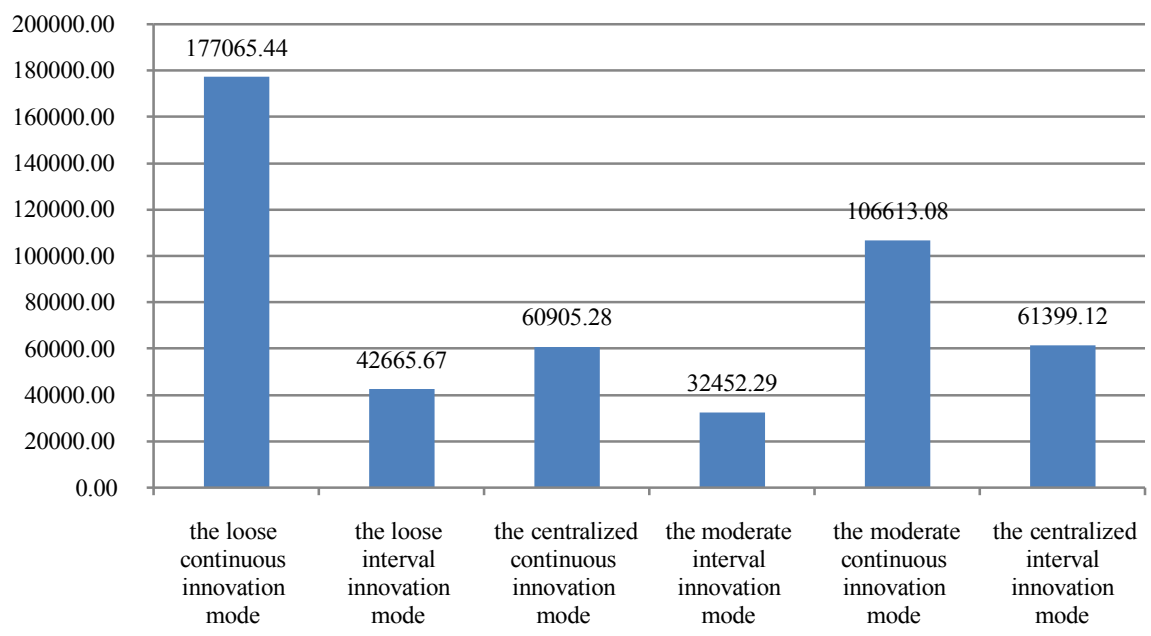

Figure 10. Business performance of different patterns of large-scale enterprises. Data Sources: CCER database and CSMAR database.

innovations, more energy and time will be invested, and R\&D activities will last longer. 
Finally, the analysis of the business performance of each pattern under different scales shows that the most efficient patterns at different scales are different. When conditions permit, small-scale, medium-scale and large-scale enterprises respectively choose the centralized continuous innovation pattern, the centralized interval innovation pattern, and the loose continuous innovation pattern, which are the most efficient innovation persistence patterns. The most efficient pattern for small-scale enterprises is also the pattern they use primarily, but medium-scale and large-scale enterprises are not the same. The main reason is that most medium-scale enterprises are in a transitional stage, the ownership structure is not completely separated, and more research time is needed from the application-based innovation to the basic innovation. Moreover, it is not innovative every year. That is why the centralized interval innovation pattern has the best benefits, but most of medium-scale enterprises are the moderate interval innovation pattern. After enterprises' scale reach large-scale enterprises, their strategic goals have shifted. They hope to become the continuous leader of the industry through major breakthroughs in transformation; it may take many years to produce innovative output. But large enterprises have more capacity and capital, so innovation output has increased to some extent. That is why most of the large-scale enterprises are the loose interval innovation pattern, but still have difficulty reaching the loose continuous innovation pattern.

Of course, this paper has the following shortcomings: Firstly, this paper takes the manufacturing enterprise as the representative to study the innovation persistence model of the enterprises. In the future, we can try to analyze and identify the innovation persistence model of other industries. Secondly, this paper only shows the recognition results of the BP neural network model. In the future, the effects of various models can be compared to further improve the results. Finally, in management practice, the specific content of the innovation persistence model of different enterprises should also be based on the characteristics of the company's managers, the policies and trends introduced by the state, the external macro environment and other factors. So in the future we will study how to build a more operational model.

\section{Conflicts of Interest}

The authors declare no conflicts of interest regarding the publication of this paper.

\section{References}

[1] Malerba, F., Orsenigo, L. and Peretto, P. (1997) Persistence of Innovative Activities, Sectoral Patterns of Innovation and International Technological Specialization. International Journal of Industrial Organization, 15, 801-826. https://doi.org/10.1016/S0167-7187(97)00012-X

[2] Raymond, W., Mohnen, P., Palm, F. and van der Loeff, S.S. (2006) Persistence of Innovation in Dutch Manufacturing: Is It Spurious? CESifo Working Paper 92, 495-504. 
[3] Huang, L.C., Luo, Y.F., Zhang, H.C. and Li, X.Y. (2006) Technology Innovation Ability Analysis of Beijing's Manufacturing Industry. IEEE International Conference on Management of Innovation \& Technology, Singapore, 21-23 June 2006, 1, 195-201.

[4] Abraham, B.P. and Moitra, S.D. (2001) Innovation Assessment through Patent Analysis. Technovation, 21, 245-252. https://doi.org/10.1016/S0166-4972(00)00040-7

[5] Abbas, A., Zhang, L. and Khan, S.U. (2014) A Literature Review on the State-of-the-Art in Patent Analysis. World Patent Information, 37, 3-13. https://doi.org/10.1016/j.wpi.2013.12.006

[6] Jensen, M.C. and Meckling, W.H. (1976) Theory of the Firm: Managerial Behavior, Agency Costs and Ownership Structure. Journal of Financial Economics, 3, 305-360. https://doi.org/10.1016/0304-405X(76)90026-X

[7] Demsetz, H. (1983) The Structure of Ownership and the Theory of the Firm. Journal of Law \& Economics, 26, 375-390. https://doi.org/10.1086/467041

[8] Francis, J. and Smith, A. (1995) Agency Costs and Innovation Some Empirical Evidence. Journal of Accounting and Economics, 19, 383-409. https://doi.org/10.1016/0165-4101(94)00389-M

[9] Lerner, J. and Wulf, J. (2007) Innovation and Incentives: Evidence from Corporate R\&D. Review of Economics \& Statistics, 89, 634-644. https://doi.org/10.1162/rest.89.4.634

[10] Ang, J.S., Cole, R.A. and Lin, J.W. (2000) Agency Costs and Ownership Structure. The Journal of Finance, 55, 81-106. https://doi.org/10.1111/0022-1082.00201

[11] Fama, E.F. and Jensen, M.C. (1983) Separation of Ownership and Control. The Journal of Law and Economics, 26, 301-325.

[12] Colombo, M.G., Croce, A. and Murtinu, S. (2014) Ownership Structure, Horizontal Agency Costs and the Performance of High-Tech Entrepreneurial Firms. Small Business Economics, 42, 265-282. https://doi.org/10.1007/s11187-013-9483-y

[13] Gogineni, S., Linn, S.C. and Yadav, P.K. (2010) Empirical Evidence on Ownership Structure, Management Control and Agency Costs. Working Paper. University of Oklahoma.

[14] Raudys, S.J. and Jain, A.K. (1991) Small Sample Size Effects in Statistical Pattern Recognition: Recommendations for Practitioners and Open Problems. IEEE Transactions on Pattern Analysis and Machine Intelligence, 13, 252-264. https://doi.org/10.1109/34.75512

[15] Jain, A.K., Duin, R.P.W. and Mao, J. (2000) Statistical Pattern Recognition: A Review. IEEE Transactions on Pattern Analysis and Machine Intelligence, 22, 4-37. https://doi.org/10.1109/34.824819

[16] Jelinek, F. (1997) Statistical Methods for Speech Recognition. Statistical Methods for Speech Recognition. MIT Press, New York.

[17] Fu, K.S. (1977) Syntactic Pattern Recognition, Applications. Springer-Verlag, Berlin, 14. https://doi.org/10.1007/978-3-642-66438-0

[18] Rengaswamy, R. and Venkatasubramanian, V. (1995) A Syntactic Pattern-Recognition Approach for Process Monitoring and Fault Diagnosis. Engineering Applications of Artificial Intelligence, 8, 35-51. https://doi.org/10.1016/0952-1976(94)00058-U

[19] Vidal, E. and Garcia, P. (2002) Inference of $K$-Testable Languages in the Strict Sense and Application to Syntactic Pattern Recognition. IEEE Transactions on Pattern Analysis and Machine Intelligence, 12, 920-925. 
[20] Pedrycz, W. (1990) Fuzzy Sets in Pattern Recognition: Methodology and Methods. Pattern Recognition, 23, 121-146. https://doi.org/10.1016/0031-3203(90)90054-O

[21] Taha, M.M.R. and Lucero, J. (2005) Damage Identification for Structural Health Monitoring Using Fuzzy Pattern Recognition. Engineering Structures, 27, 1774-1783. https://doi.org/10.1016/j.engstruct.2005.04.018

[22] Fulkerson, B. (1995) Machine Learning, Neural and Statistical Classification. Technometrics, 37, 459. https://doi.org/10.1080/00401706.1995.10484383

[23] Neal, R.M. (1996) Bayesian Learning for Neural Networks. Springer, Berlin.

[24] Abirami, S. and Neelamegam, P.H.K. (2014) Analysis of Rice Granules Using Image Processing and Neural Network Pattern Recognition Tool. International Journal of Computer Applications, 96, 20-24. https://doi.org/10.5120/16806-6530

[25] Pal, S.K., Ray, S.S. and Ganivada, A. (2017) Granular Neural Networks, Pattern Recognition and Bioinformatics. In: Studies in Computational Intelligence, Vol. 712, Springer, Berlin.

[26] Fu, J., Li, G., Qin, Y. and Freeman, W.J. (2007) A Pattern Recognition Method for Electronic Noses Based on an Olfactory Neural Network. Sensors and Actuators B: Chemical, 125, 489-497. https://doi.org/10.1016/j.snb.2007.02.058

[27] Mirfakhraei, K. and Horch, K. (1994) Classification of Action Potentials in Multi-Unit Intrafascicular Recordings Using Neural Network Pattern-Recognition Techniques. IEEE Transactions on Biomedical Engineering, 41, 89-91. https://doi.org/10.1109/10.277276

[28] Mcculloch, W.S. and Pitts, W. (1990) A Logical Calculus of the Ideas Immanent in Nervous Activity. Bulletin of Mathematical Biology, 52, 99-115. https://doi.org/10.1007/BF02459570

[29] Rosenblatt, F. (1958) The Perceptron: A Probabilistic Model for Information Storage and Organization in the Brain. Psychological Review, 65, 386-408. https://doi.org/10.1037/h0042519

[30] Hopfield, J.J. (1982) Neural Networks and Physical Systems with Emergent Collective Computational Abilities. Proceedings of the National Academy of Sciences of the United States of America, 79, 2554-2558. https://doi.org/10.1073/pnas.79.8.2554

[31] Rumelhart, D.E., Hinton, G.E. and Williams, R.J. (1986) Learning Representations by Back-Propagating Errors. In: Anderson, J.A. and Rosenfeld, E., Eds., Neurocomputing. Foundations of Research, MIT Press, Cambridge, 533-536. https://doi.org/10.1038/323533a0

[32] Ackley, D.H., Hinton G.E. and Sejnowski, T.J. (1985): A Learning Algorithm for Boltzmann Machines. Cognitive Science, 9, 147-169. https://doi.org/10.1207/s15516709cog0901_7

[33] Werbos, P.J. (1994) The Roots of Backpropagation: From Ordered Derivatives to Neural Networks and Political Forecasting. Wiley, New York.

[34] Rumelhart, D.E., Hinton, G.E. and Williams, R.J. (1988) Learning Internal Representations by Error-Propagation. Readings in Cognitive Science, 323, 399-421.

[35] Jain, A. and Zongker, D. (1997) Feature Selection: Evaluation, Application, and Small Sample Performance. IEEE Transactions on Pattern Analysis and Machine Intelligence, 19, 153-158. https://doi.org/10.1016/S1088-467X(97)00008-5

[36] Dash, M. and Liu, H. (1997) Feature Selection for Classification. Intelligent Data Analysis, 1, 131-156. https://doi.org/10.3233/IDA-1997-1302 
[37] Li, J., Cheng, K. and Wang, S., et al. (2016) Feature Selection: A Data Perspective. ACM Computing Surveys, 50, Article No. 94.

[38] Yin, L.Z. and Liu, H. (2009) Feature Selection for High-Dimensional Data. Computational Management Science, 6, 25-40. https://doi.org/10.1007/s10287-008-0070-7 